\title{
Estudo Comparativo entre Bupivacaína a 0,5\% e Mistura Enantiomérica de Bupivacaína (S75-R25) a 0,5\% em Anestesia Peridural *
}

\section{Comparative Study between 0.5\% Bupivacaine and 0.5\% Enantiomeric Mixture of Bupivacaine (S75-R25) in Epidural Anesthesia}

Rosane Fossatti Gonçalves ${ }^{1}$, Gabriela Rocha Lauretti ${ }^{2}$, Anita Leocádia de Mattos, TSA ${ }^{3}$

\begin{abstract}
RESUMO
Gonçalves RF, Lauretti GR, Mattos AL - Estudo Comparativo entre Bupivacaína a 0,5\% e Mistura Enantiomérica de Bupivacaína (S75-R25) a 0,5\% em Anestesia Peridural
\end{abstract}

Justificativa e Objetivos - A mistura enantiomérica de bupivacaína (S75-R25) vem sendo empregada pela sua propriedade anestésica com menor toxicidade do que a bupivacaína racêmica. O objetivo deste estudo é comparar a bupivacaína a $0,5 \%$ com a mistura enantiomérica de bupivacaína a 0,5\% (S75-R25) em anestesia peridural.

Método - Foram incluídos no estudo 44 pacientes divididos em dois grupos $(n=22)$ denominados de Bupivacaína e S75-R25. Os pacientes foram medicados com midazolam por via venosa. A anestesia peridural foi realizada no espaço $L_{3}-L_{4}$ ou $L_{2}-L_{3}$, e administrado 16 a $24 \mathrm{ml}$ da solução do anestésico local. O grupo Bupivacaína recebeu bupivacaína a 0,5\% com vasoconstritor. O grupo S75-R25 recebeu a mistura enantiomérica de bupivacaína a $0,5 \% \mathrm{com}$ vasoconstritor. Foram avaliados a temperatura do membro inferior antes e após o bloqueio peridural, o tempo de latência do bloqueio, o tipo de alteração referida pelo paciente, possíveis falhas sensoriais, nível sensorial metamérico e o grau de bloqueio motor. Na sala de recuperação pós-anestésica, foi anotado o tempo de requisição do primeiro analgésico.

Resultados - Fizeram parte da avaliação final 41 pacientes. Os grupos foram demograficamente semelhantes. A dose per-operatória de midazolam, o volume de anestésico local por via peridural, o tempo de latência para a instalação do bloqueio, falhas sensoriais a picada da agulha, temperatura do membro inferior nos diferentes tempos, o tipo de sensação parestésica, e o nível anestésico em dermátomos foram semelhantes entre os grupos. O grau de bloqueio motor foi mais intenso para o grupo Bupivacaína, comparado ao grupo S75-R25 ( $p=0,0117)$. O tempo para requisição do primeiro analgésico no período pós-operatório foi superior para o grupo S75-R25, comparado ao grupo Bupivacaína (596 \pm 436 minutos versus $463 \pm 270$ minutos, respectivamente;

\footnotetext{
* Recebido do (Received from) Hospital das Clínicas da Faculdade de Medicina de Ribeirão Preto - Universidade de São Paulo (HCFMRP USP), Ribeirão Preto, $S P$

1. Pós-graduanda, Anestesiologista da FMRP-USP

2. Professora Doutora Livre Docente do Departamento de Biomecânica, Medicina e Reabilitação do Aparelho Locomotor- FMRP-USP

3. Professora Doutora do Departamento de Biomecânica, Medicina e Reabilitação do Aparelho Locomotor- FMRP-USP
}

Apresentado (Submitted) em 23 de maio de 2002

Aceito (Accepted) para publicação em 20 de agosto de 2002

Correspondência para (Mail to):

Dra. Gabriela Rocha Lauretti

Rua Campos Sales, 330/44

14015-110 Ribeirão Preto, SP

(C) Sociedade Brasileira de Anestesiologia, 2003 $p=0,04572)$. A incidência de efeitos adversos foi semelhante entre os grupos.

Conclusões - A mistura enantiomérica de bupivacaína (S75-R25) apresentou maior tempo analgésico e menor grau de bloqueio motor, comparada com a solução de bupivacaína racêmica.

UNITERMOS: ANESTÉSICOS, Local: bupivacaína, mistura enantiomérica de bupivacaína (S75-R25); TÉCNICAS ANESTÉSICAS, Regional: peridural

\section{SUMMARY}

Gonçalves RF, Lauretti GR, Mattos AL - Comparative Study between $0.5 \%$ Bupivacaine and $0.5 \%$ Enantiomeric Mixture of Bupivacaine (S75-R25) in Epidural Anesthesia

Background and Objectives - A bupivacaine formulation containing $25 \%$ of $R(+)$ and $75 \%$ of $S(-)$ isomer has been used because its anesthetic properties with less toxicity than the racemic bupivacaine. This study aimed at evaluating the racemic bupivacaine as compared to B25/75 in epidural anesthesia.

Methods - Participated in this study 44 patients who were distributed in two groups $(n=22)$, namely Bupivacaine and S75-R25. Patients were premedicated with intravenous midazolam. Epidural anesthesia was induced at $L_{3}-L_{4}$ or $L_{2}-L_{3}$ interspace with 16 to $24 \mathrm{ml}$ of the anesthetic solution. Group Bupivacaine received $0.5 \%$ bupivacaine with vasoconstrictor. Group S75-R25 received the enantiomeric mixture of $0.5 \%$ bupivacaine with vasoconstrictor. The following parameters were evaluated: lower limb temperature before and after epidural block, blockade onset, type of sensation referred by the patient, possible sensory failures, metameric sensory level and motor block level. Time for first analgesic request in the PACU was also recorded.

Results - Forty-one patients completed the study. Groups were demographically similar. Perioperative midazolam dose, epidural anesthetic volume, blockade onset, sensory failures at pinprick, lower limb temperature in different moments, type of paresthesia sensation and anesthetic depth in dermatomes were similar between groups. Motor block was less intense in group S75-R25 ( $p=0.0117)$ as compared to group Bupivacaine. Time to first postoperative analgesic dose was longer for group S75-R25 as compared to group Bupivacaine $(596 \pm 436 \mathrm{~min}$ versus $463 \pm 270 \mathrm{~min}$, respectively; $p=$ 0.04572). The incidence of adverse effects was similar between groups.

Conclusions - Enantiomeric bupivacaine (S75-R25) resulted in longer analgesia and less intense motor block as compared to racemic bupivacaine.

KEY WORDS: ANESTHETICS, Local: bupivacaine, enantiomeric mixture of bupivacaine (S75-R25); ANESTHETIC TECHNIQUES, Regional: epidural 


\section{INTRODUÇÃO}

$\mathrm{R}_{\mathrm{i}}^{\mathrm{s}}$ elatos de cardiotoxicidade resultante da administração inadvertida de bupivacaína racêmica por via venosa estimularam a pesquisa e o desenvolvimento de formulações alternativas oriundas da fórmula estrutural básica ${ }^{1-3}$. O isômero isolado S(-)bupivacaína (levobupivacaína) demonstrou ser menos tóxico aos sistemas nervoso central e cardiovascular $^{1,4}$, preservando as propriedades da formulação racêmica na anestesia periférica, como na anestesia infiltrativa, anestesia do plexo braquial, bloqueio ileoinguinal/hipogástrico e bloqueio do nervo ciático ${ }^{5,6}$.

Embora a levobupivacaína apresente eficácia anestésica em bloqueios periféricos, dados sobre a administração por via espinhal são conflitantes ${ }^{5,7-10}$. A administração peridural de levobupivacaína está associada à menor intensidade de bloqueio motor $^{5,9,10}$, sendo aparentemente dose-dependente. Doses analgésicas variaram de $0,0625 \%$ a $0,5 \%{ }^{5,11}$, enquanto a levobupivacaína a $0,75 \%$ foi equivalente à formulação racêmica em relação à intensidade dos bloqueios motor e sensitivo ${ }^{12}$.

Uma nova formulação contendo $25 \%$ do isômero $\mathrm{R}(+)$ e $75 \%$ do isômero S(-)-bupivacaína, resultou em anestesia comparável à formulação racêmica, quando administrada por via subaracnóidea ${ }^{13}$, sugerindo que a adição de $25 \%$ do isômero $\mathrm{R}(+)$-bupivacaína melhoraria o perfil anestésico da droga, sem contudo aumentar a incidência dos efeitos tóxicos. Este estudo visou avaliar a eficácia anestésica da mistura enantiomérica de bupivacaína (S75-R25) quando administrada por via peridural, comparada a bupivacaína racêmica (S50-R50).

\section{MÉTODO}

O estudo foi aprovado pela Comissão de Normas Éticas do Hospital das Clínicas da Faculdade de Medicina de Ribeirão Preto - Universidade de São Paulo, e foi obtido o consentimento pós-informação para participação. Participaram 44 pacientes, com idades entre 18 e 65 anos, estado físico ASAI ou II, de ambos os sexos, submetidos a procedimentos cirúrgicos vasculares ou ortopédicos, no membro inferior. O estudo foi prospectivo, aleatório, duplamente encoberto e os pacientes foram divididos em dois grupos $(n=22)$ : grupo Bupivacaína e grupo S75-R25.

Todos os pacientes fizeram uso de midazolam $(0,05$ a 0,1 $\mathrm{mg} \cdot \mathrm{kg}^{-1}$ ) por via venosa, na sala de recepção anestésica, após punção venosa em um dos membros superiores com cateter venoso 20G. A hidratação per-operatória constou de solução de Ringer com lactato, sendo o total $15 \mathrm{ml} . \mathrm{kg}^{-1}$.

Na sala cirúrgica, antes do início da anestesia, foram realizadas três medidas consecutivas das pressões arteriais sistólica, diastólica e média com aparelho de leitura não-invasivo a intervalos de 5 minutos. Esta monitorização continuou a intervalos de 3 minutos durante os primeiros 21 minutos, e 5 minutos, após. A freqüência cardíaca (traçado eletrocardiográfico) e a saturação de $\mathrm{O}_{2}\left(\mathrm{SpO}_{2}\right)$ avaliada através de oximetria de pulso foram medidas contínuas. A temperatura do membro inferior foi avaliada em dois momentos, através de termômetro aderido ao dorso do pé, antes da realização do bloqueio peridural (momento zero) e 30 minutos após a realização do bloqueio peridural (momento 30 ).

A anestesia peridural foi realizada com o paciente sentado, nos espaços $L_{3}-L_{4}$ ou $L_{2}-L_{3}$. Após a dose teste para saber do correto posicionamento da agulha, com lidocaína a 1\% (3 ml) com vasoconstritor, foram aguardados três minutos para exclusão de punção inadvertida venosa ou subaracnóidea. Foram administrados 16 a $24 \mathrm{ml}$ do anestésico local em bolus (60 segundos), o qual estava em frasco ampola codificado, a critério do anestesiologista. Após a punção peridural, o paciente permaneceu em decúbito dorsal. O grupo Bupivacaína recebeu bupivacaína a $0,5 \%$ racêmica, com vasoconstritor. O grupo S75-R25 recebeu a mistura enantiomérica de bupivacaína a $0,5 \%$, com vasoconstritor.

Foi anotado o tempo de latência desde a punção peridural até que o paciente referiu sensação de parestesia. O tipo de alteração referida pelo paciente, como sensação de peso, formigamento, dormência ou aumento da temperatura foram anotados. Após 30 minutos, foram avaliadas as possíveis faIhas de analgesia e anotadas como ausência de falha e falha parcial (envolveu apenas 1 metâmero, abaixo do nível de anestesia), porém não necessitou sedação venosa adicional; ou falha total (envolveu mais de um metâmero abaixo do nível de dermátomo da anestesia, e necessitou sedação com propofol como complementação da técnica anestésica). Em 5, 10, 15, 20 e 30 minutos, foi avaliado o nível sensorial em dermátomos, utilizando-se o teste à picada da aguIha, feito delicadamente, e sem lesar a pele, no abdômen do paciente na região anestesiada e não anestesiada.

O grau de bloqueio motor para cada paciente foi avaliado 30 minutos após a punção peridural sempre pelo mesmo anestesiologista, e classificado de acordo com a escala de Bromage como: 1) ruim (3);2) bom (2);3) ótimo (1), e 4) excelente $(0)$.

Quaisquer efeitos adversos ocorridos durante o período peri-operatório foram anotados. Após o término do procedimento cirúrgico, os pacientes permaneceram na sala de recuperação pós-anestésica até o momento da requisição do primeiro analgésico. O tempo desde a punção peridural até este momento foi anotado. O valor numérico da intensidade da dor neste momento foi avaliado utilizando-se a Escala Analógica Visual de $10 \mathrm{~cm}$, sendo o extremo "zero cm" correspondente a "ausência de dor", variando até "dez cm", correspondente à "pior dor imaginável". Os pacientes foram medicados com $100 \mathrm{mg}$ de cetoprofeno por via venosa, e considerou-se o término da avaliação.

O número mínimo de pacientes determinado pelo teste do poder estatístico foi baseado em estudo preliminar realizado em nosso serviço, considerando alfa $=5 \%$ e beta $=0,1$. Os grupos foram comparados para análise demográfica, nível de bloqueio, temperatura entre dois grupos, tempo de latência, tempo de requisição do primeiro analgésico, valor numérico para intensidade da dor durante a requisição do primeiro analgésico, utilizando-se o teste não paramétrico de Mann-Whitney U. Os valores de temperatura em um mesmo 
grupo nos diferentes tempos foram avaliados utilizando-se o teste pareado de Wilcoxon. Os dados foram expressos como média \pm desvio padrão. Incidência de efeitos adversos, sexo, tipo de sensação parestésica pós-bloqueio, falha e grau de bloqueio motor foram comparados entre os grupos utilizando o teste Qui-quadrado, sendo que $p<0,05$ foi considerado significante. Os valores de pressão arterial, pulso e oximetria foram comparados utilizando-se ANOVA bi-caudal para medidas repetidas. Foi considerado significativo $p<0,05$.

\section{RESULTADOS}

Quarenta e um pacientes fizeram parte da avaliação final. Três dos pacientes do grupo bupivacaína foram excluídos devido a dados incompletos. Dos vinte e dois pacientes do grupo S75-R25, 17 foram submetidos à safenectomia e 5 foram submetidos a procedimentos ortopédicos de pequeno porte na tíbia. Dos 19 pacientes do grupo Bupivacaína, 15 foram submetidos à safenectomia, enquanto 4 submetidos a procedimentos ortopédicos de pequeno porte na tíbia ( $p=$ 0,2576 ). Os dois grupos foram demograficamente semeIhantes em relação ao estado físico, sexo, idade, estatura e peso (Tabela I).

Tabela I - Dados Demográficos

\begin{tabular}{lcc}
\hline & Grupo Bupivacaína & Grupo S75-R25 \\
\hline Idade $(\mathrm{anos})^{*}$ & $46 \pm 13$ & $46 \pm 14$ \\
Altura $(\mathrm{cm})^{*}$ & $162 \pm 8$ & $166 \pm 10$ \\
Peso $(\mathrm{kg})^{*}$ & $72 \pm 13$ & $71 \pm 14$ \\
Sexo & & \\
$\quad$ Masculino & 3 & 7 \\
$\quad$ Feminino & 16 & 15 \\
Estado físico & & \\
$\quad$ ASA I & 8 & 7 \\
ASA II & 11 & 15 \\
\hline
\end{tabular}

* Valores expressos pela Média \pm DP

A dose final de midazolam utilizada durante o período peri-operatório, e o volume de anestésico local utilizado no espaço peridural nos grupos Bupivacaína e S75-R25, respectivamente, foram semelhantes (midazolam - 4,4 $\pm 3 \mathrm{mg}$; $4,8 \pm 2 \mathrm{mg}$; volume de anestésico local $-20 \pm 2 \mathrm{ml} ; 19,5 \pm 4 \mathrm{ml}$ ). Os valores das pressões arteriais sistólica, diastólica e média, freqüência cardíaca e saturação de $\mathrm{O}_{2}\left(\mathrm{SpO}_{2}\right)$ foram semelhantes entre os grupos quando avaliados em intervalos constantes $(p>0,05)$.

Atemperatura do membro inferior antes da realização do bloqueio peridural e 30 minutos após a realização do bloqueio peridural está descrita na tabela II. A avaliação entre os dois grupos nos tempos "zero" e "30 minutos" após a injeção peridural não demonstrou diferença estatística ( $p>0,05)$, enquanto que a avaliação em um mesmo grupo nos tempos "zero" e "30 minutos", foi diferente $(p<0,005)$.
Tabela II - Temperatura $\left({ }^{\circ} \mathrm{C}\right)$ do Membro Inferior nos Diferentes Tempos

\begin{tabular}{lcc}
\hline & Grupo Bupivacaína & Grupo S75-R25 \\
\hline Tempo zero & $33,2 \pm 1,4$ & $33 \pm 1,5$ \\
Tempo $30 \mathrm{~min}$ & $34,4 \pm 1,2$ & $34,1 \pm 1,5$ \\
\hline
\end{tabular}

O tempo de latência desde a injeção peridural até que o paciente referiu sensação de parestesia foi semelhante entre os grupos (Bupivacaína - 6,2 \pm 3,2 min; S75-R25- 8,2 \pm 6,2 min). Os tipos de alterações (sensação de peso, formigamento, dormência, ou aumento da temperatura) foram semelhantes entre os grupos, e está descrito na tabela III. O nível sensorial metamérico aos $5 \mathrm{~min}$ (T11 a T12), aos 10 $\min$ (T9), aos $15 \mathrm{~min}$ (T8), aos $20 \mathrm{~min}$ (T7 a T8) e aos $30 \mathrm{~min}$ (T7 a T8) foram igualmente semelhante entre os grupos $(p>0,05)$.

Tabela III - Número de Pacientes em Cada Grupo que Referiu as Diferentes Sensações Parestésicas em Membros Inferiores, após a Administração do Anestésico Local por Via Peridural

\begin{tabular}{lcc}
\hline & Grupo Bupivacaína & Grupo S75-R25 \\
\hline Dormência & $12^{*}$ & $17^{*}$ \\
Peso & 3 & 3 \\
Formigamento & 2 & 1 \\
Aumento de temperatura & 2 & 1 \\
\hline$*$
\end{tabular}

* $p<0,05$

Em relação ao grau de falha do bloqueio, avaliada 30 minutos após a injeção peridural, no grupo S75-R25, um paciente apresentou falha parcial e outro paciente apresentou falha total, necessitando de sedação venosa com infusão contínua de propofol. No grupo Bupivacaína, nenhum dos pacientes apresentou falha sensorial $(p=0,2875)$. O grau de bloqueio motor foi mais intenso para o grupo Bupivacaína, comparado ao grupo S75-R25 (Tabela IV) ( $p=0,0117$ ). O tempo para requisição do primeiro analgésico no período pós-operatório foi superior para o grupo $575-\mathrm{R} 25$, comparado ao grupo Bupivacaína ( $596 \pm 436$ minutos versus $463 \pm 270$ minutos, respectivamente), sendo que os valores numéricos da escala analógica visual neste momento foram semelhantes

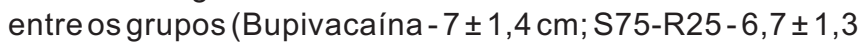
$\mathrm{cm})$.

Tabela IV - Grau de Bloqueio Motor Classificado pelo Anestesiologista

\begin{tabular}{lcc}
\hline & Grupo Bupivacaína & Grupo S75-R25 \\
\hline Ruim & $0 *$ & $2 *$ \\
Bom & $0 *$ & $3 *$ \\
Ótimo & 4 & 4 \\
Excelente & 15 & 13 \\
\hline
\end{tabular}

* $p<0,05$ 
Em relação a efeitos adversos durante o período per-operatório, 4 pacientes do grupo Bupivacaína apresentaram hipotensão arterial, sendo medicados com efedrina por via venosa; um paciente apresentou tremor, um paciente apresentou bradicardia, não sendo necessário tratamento farmacológico, e um paciente apresentou náusea. No grupo S75-R25, três pacientes apresentaram hipotensão arterial, sendo tratados com efedrina por via venosa, um paciente apresentou tremor e o paciente que apresentou falha do bloqueio foi submetido à sedação por via venosa com propofol contínuo $(p>$ $0,05)$. Não foram observados efeitos adversos no período pós-operatório em nenhum dos grupos.

\section{DISCUSSÃO}

A literatura relata que a administração por via peridural do isômero isolado S(-)-bupivacaína (levobupivacaína) apresentou menor incidência de bloqueio motor, e maior tempo de analgesia pós-operatória, sendo dose-dependente ${ }^{5,12}$. Somente a solução a $0,75 \%$ de levobupivacaína por via peridural foi semelhante à bupivacaína racêmica em relação ao tempo de latência do bloqueio sensitivo, qualidade e duração do bloqueio motor ${ }^{13}$. A hipótese de que uma formulação contendo $25 \%$ do isômero $R(+)$-bupivacaína e $75 \%$ do isômero S(-)-bupivacaína melhoraria o perfil anestésico da fórmula foi avaliada por via subaracnóidea, sendo as duas formulações semelhantes em relação à potência anestésica ${ }^{14}$. Entretanto, os resultados obtidos neste estudo por via peridural demonstraram que a adição de $25 \%$ do isômero $\mathrm{R}(+)$-bupivacaína, associado a $75 \%$ do isômero S(-)-bupivacaína a $0,5 \%$, resultou em menor intensidade de bloqueio motor, comparada com a solução racêmica. Um benefício foi o maior tempo de analgesia pós-operatória observado com a mistura enantiomérica, comparada com a bupivacaína racêmica. Os outros parâmetros avaliados, como tipo de sensação parestésica, falhas de bloqueio sensoriais, efeitos adversos provenientes da administração peridural, temperatura, foram semelhantes entre os dois grupos.

Quando comparada com dados da literatura, a mistura enantiomérica S75-R75 comportou-se aparentemente de forma semelhante à levobupivacaína pura, em relação ao bloqueio motor $^{5,10,11}$, enquanto uma concentração maior de levobupivacaína a $0,75 \%$ seria similar à formulação racêmica como anestésico ${ }^{13}$. Quando levobupivacaína a $0,75 \%$ e bupivacaína racêmica a $0,75 \%$ foram utilizadas por via peridural para cirurgias do abdômen inferior, a qualidade do bloqueio motor foi avaliada pelo cirurgião e pelo anestesiologista, sendo que, enquanto o cirurgião considerou as duas formulações idênticas, o anestesiologista foi tendencioso a quantificar o bloqueio motor da solução racêmica como qualitativamente superior ao da levobupivacaína, embora não fosse estatisticamente significante $(p=0,07)^{13}$.

No estudo atual, a mesma anestesiologista foi a responsável pela colheita dos dados sobre bloqueio motor, averiguados por um segundo co-autor, obtendo concordância de ambos. Apesar da anestesiologista classificar a intensidade do bloqueio motor como inferior (Bromage 2 e 3), a técnica anesté- sica não foi prejudicada em $95 \%$ dos pacientes do grupo S75-R25, para o tipo de procedimento do estudo (safenectomia e cirurgias ortopédicas de pequeno porte de tíbia). Se a qualidade de bloqueio motor obtido prejudicaria cirurgias intra-abdominais, não pode ser aferida deste estudo.

O interesse clínico para a procura de novas e mais eficientes formulações anestésicas de longa duração reside no fato de a toxicidade do sistema cardiovascular e sistema nervoso central encontrar-se na forma isomérica $R(+)$-bupivacaína 1,4,5,15. A incidência de intoxicação por anestésicos locais é rara, porém catastrófica. Anestésicos locais bloqueiam os canais de sódio periféricos, rápidos, voltagem-dependentes, dos axônios e estas drogas têm uma alta afinidade pelo sistema nervoso central e sistema cardiovascular. A toxicidade do sistema nervoso central segue um processo de dois estágios, que quando em baixas concentrações, promove o bloqueio de neurônios inibitórios, resultando em convulsões generalizadas; enquanto em altas concentrações, ocorre uma depressão global do sistema nervoso central. Apesar das crises convulsivas serem clinicamente impressionantes, podem habitualmente ser tratadas sem resultar em lesão permanente. O mais importante é a cardiotoxicidade, que pode ser mediada indiretamente pelo cérebro e diretamente no miocárdio. De forma semelhante ao sistema nervoso central, a cardiotoxicidade indireta ocorre inicialmente como ação estimulatória, decorrente de concentrações mais baixas, seguida de um componente depressivo em concentrações maiores. As ações diretas no miocárdio incluem inotropismo, cronotropismo e dromotropismo negativos. Aestereosseletividade é encontrada para o dromotropismo. O isômero S(-)bupivacaína produziu um menor atraso na condução, comparado à formulação racêmica ${ }^{16}$. A dose letal em $50 \%$ dos animais (camundongos e ratos) foi $30 \%-40 \%$ mais baixa para o isômero $\mathrm{R}(+)$-comparado com o isômero $\mathrm{S}(-)$ bupivacaína ${ }^{6}$. Acardiotoxicidade reside no fato de a forma $\mathrm{R}(+)$ possuir maior afinidade e se dissociar mais lentamente dos canais iônicos, predispondo às disritmias cardíacas ${ }^{4}$. Em cães anestesiados, a taxa de mortalidade foi $20 \%$ maior para a formulação racêmica comparada à levobupivacaína ${ }^{17}$. Aidéia de se obter um anestésico local de longa duração que apresente boa qualidade de anestesia e analgesia, comparável à solução padrão de bupivacaína racêmica, com menor toxicidade, é tentadora. Alternativas que mereceriam meIhor avaliação seriam o isômero puro $\mathrm{S}(-)$ - bupivacaína a $0,75 \%$, ou a S75-R25 em maior concentração, a fim de manter a qualidade do bloqueio motor e de ampliar o grau de analgesia. A adição do isômero R(-)-bupivacaína a $25 \%$ poderia trazer benefícios teóricos, como maior potência antibacteriana contra Staphylococcus aureus, S epidermidis, Enterococcus faecalis ${ }^{18}$. Resta saber se a adição de $25 \%$ do $\mathrm{R}(+)$-bupivacaína à formulação seria mais vantajosa quando comparada com a solução levobupivacaína a 0,75\%, sendo equipotentes tanto quanto à anestesia, quanto à incidência dos efeitos tóxicos.

Pelos dados obtidos, pode-se concluir que, nas condições deste estudo, a mistura enantiomérica de bupivacaína (S75-R25) apresentou maior tempo de analgesia e menor 
grau de bloqueio motor quando comparada à bupivacaína racêmica (S50-R50).

\section{Comparative Study between $0.5 \%$ Bupivacaine and $0.5 \%$ Enantiomeric Mixture of Bupivacaine (S75-R25) in Epidural Anesthesia}

Rosane Fossatti Gonçalves, M.D., Gabriela Rocha Lauretti, M.D., Anita Leocádia de Mattos, TSA, M.D.

\section{INTRODUCTION}

Reports on cardiotoxicity resulting from the inadvertent intravenous administration of racemic bupivacaine have encouraged research and development of alternative techniques arising from the basic structural formula ${ }^{1-3}$. The isolated isomer S(-) bupivacaine (levobupivacaine) was shown to be less toxic to central nervous and cardiovascular systems ${ }^{1,4}$, however preserving racemic formulation properties in peripheral anesthesia, such as infiltrative anesthesia, brachial plexus anesthesia, ileo-inguinal/hypogastric blockade and sciatic nerve blockade ${ }^{5,6}$.

Although being effective for peripheral blocks, data on spinal levobupivacaine are controversial 5,7-10. Epidural levobupivacaine is associated to less intense motor block $5,9,10$ and is seemingly dose-dependent. Analgesic doses vary from $0.0625 \%$ to $0.5 \%{ }^{5,11}$, while $0.75 \%$ levobupivacaine is equivalent to the racemic formulation in motor and sensory block intensity ${ }^{12}$.

Anew formulation with $25 \%$ of isomer $\mathrm{R}(+)$ and $75 \%$ of isomer $\mathrm{S}(-)$ bupivacaine resulted in spinal anesthesia equivalent to the racemic formulation ${ }^{13}$ suggesting that the addition of $25 \%$ of isomer $R(+)$ bupivacaine would improve drug's anesthetic profile without however increasing the incidence of toxic effects. This study aimed at evaluating the anesthetic efficacy of epidural S75-R25 bupivacaine as compared to racemic bupivacaine (S50-R50).

\section{METHODS}

After the Ethical Standards Committee, Hospital das Clinicas, Faculdade de Medicina, Ribeirão Preto Universidade de São Paulo approval and their informed consent, participated in this prospective, randomized and double-blind study 44 patients of both genders, aged 18 to 65 years, physical status ASA I or II, submitted to lower limb vascular or orthopedic procedures. Patients were distributed in two groups $(n=22)$ : group Bupivacaine and group S75-R25. Patients were premedicated with intravenous midazolam ( 0.05 to $\left.0.1 \mathrm{mg} . \mathrm{kg}^{-1}\right)$ after upper limb venous puncture with a $20 \mathrm{G}$ catheter. Perioperative hydration was achieved with lactated Ringer's solution in a total of $15 \mathrm{ml} . \mathrm{kg}^{-1}$.
In the operating room and before anesthetic induction, three consecutive measurements were obtained from systolic, diastolic and mean blood pressure with non-invasive equipment at 5-minute intervals. Monitoring was maintained at 3-minute intervals during the first 21 minutes and at 5-minute intervals there after. Heart rate (ECG) and $\mathrm{O}_{2}$ saturation $\left(\mathrm{SpO}_{2}\right)$ evaluated by pulse oximetry were continuously measured. Lower limb temperature was evaluated in two moments, through a thermometer fixed to the dorsum pedis: before epidural block (moment 0 ) and 30 minutes after epidural block (moment 30 ). Epidural anesthesia was induced in the sitting position in $L_{3}-L_{4}$ or $L_{2}-L_{3}$ interspace. After a test dose with $1 \%$ lidocaine $(3 \mathrm{ml})$ and vasoconstrictor to check the right position of the needle, three minutes were waited to rule out inadvertent venous or spinal puncture. Bolus local anesthetics (16 to $24 \mathrm{ml}$ ) were administered in 60 seconds. Drugs were packaged in coded vials and were administered at anesthesiologist's judgment. After epidural puncture, patients remained in the supine position. Group Bupivacaine received $0.5 \%$ racemic bupivacaine with vasoconstrictor. Group S75-R25 received enantiomeric $0.5 \%$ bupivacaine with vasoconstrictor.

Time ellapsed from epidural puncture to patients referring paresthesia was recorded. Type of sensation referred by patients, such as heaviness, tingling, dumbness or increased temperature, were also recorded. Possible analgesic failures after 30 minutes were evaluated and recorded as no failure or partial failure (involving 1 metamer only, below anesthetic level) with no need for additional intravenous sedation; or total failure (involving more than 1 metamer below anesthetic level) and needing additional sedation with propofol. Sensory level in dermatomes through pinprick test gently applied without injuring the skin was evaluated in 5, 10, 15, 20 and 30 minutes, in patients' anesthetized and non-anesthetized abdominal regions.

Motor block was evaluated by the same anesthesiologist 30 minutes after epidural puncture, and was classified through Bromage's scale as: 1) poor (3); 2) good (2); 3) very good (1); 4) excellent (0).

Adverse effects were recorded. At surgery completion, patients remained in the post-anesthetic recovery unit until first analgesic request. Time from puncture to this moment was recorded. Pain intensity at this moment was evaluated by the Visual Analog Scale (VAS) being zero "no pain" and 10 "the worst possible pain". Patients were medicated with $100 \mathrm{mg}$ intravenous ketoprofen and evaluation was considered completed.

Minimum number of patients determined by statistical power test was based on a previous study carried out by our institution and considered alpha $=5 \%$ and beta $=0.1$. Mann-Whitney $U$ non-parametric test was used to compare demographics, blockade level, temperature, onset, time for first analgesic request and pain intensity at first analgesic request between groups. Wilcoxon paired test was used to evaluate temperature in a same group in different moments. Data are expressed in mean \pm standard deviation. Chi-square test was used to compare adverse effects, gender, type of paresthesia sensation after blockade, motor block failure 
and level between groups being considered significant $p<$ 0.05 . Two-tailed ANOVA for repeated measures was used to compare blood pressure and pulse oximetry, considering significant $p<0.05$.

\section{RESULTS}

Forty-one patients completed the study. Three group Bupivacaine patients were excluded due to incomplete data. From 22 group S75-R25 patients, 17 were submitted to saphenectomy and 5 were submitted to minor tibial orthopedic surgeries. From 19 group Bupivacaine patients, 15 were submitted to saphenectomy and 4 were submitted to minor tibial orthopedic procedures $(p=0.2576)$. Both groups were similar in gender, age, height, weight and physical status ( Table I).

Table I - Demographics Data

\begin{tabular}{lcc}
\hline & Group Bupivacaine & Group S75-R25 \\
\hline Age (years) * & $46 \pm 13$ & $46 \pm 14$ \\
Height $(\mathrm{cm})^{*}$ & $162 \pm 8$ & $166 \pm 10$ \\
Weight $(\mathrm{kg})^{*}$ & $72 \pm 13$ & $71 \pm 14$ \\
Gender & 3 & 7 \\
Male & 16 & 15 \\
Female & & \\
Physical status & 8 & 7 \\
ASA I & 11 & 15 \\
ASA II & & \\
\hline
\end{tabular}

*Values expressed in Mean \pm SD

Final perioperative midazolam dose and local anesthetic volume injected in the epidural space were similar for both groups (midazolam - $4.4 \pm 3 \mathrm{mg} ; 4.8 \pm 2 \mathrm{mg}$; local anesthetic volume - $20 \pm 2 \mathrm{ml} ; 19.5 \pm 4 \mathrm{ml}$ ). Systolic, diastolic and mean blood pressure, heart rate and oxygen saturation $\left(\mathrm{SpO}_{2}\right)$ were similar between groups when evaluated at constant intervals $(p>0.05)$

Lower limb temperature before epidural block and 30 minutes after epidural block is shown in table II. Evaluation between groups in moments "zero" and "30 minutes" after epidural injection has not shown statistical significance $(p>0.05)$, while evaluation of moments "zero" and "30 minutes" in a same group was different $(p<0.005)$.

Table II - Lower Limb Temperature $\left({ }^{\circ} \mathrm{C}\right)$ in Differents Moments

\begin{tabular}{lcc}
\hline & Group Bupivacaine & Group S75-R25 \\
\hline Moment zero & $33.2 \pm 1.4$ & $33 \pm 1.5$ \\
Moment $30 \mathrm{~min}$ & $34.4 \pm 1.2$ & $34.1 \pm 1.5$ \\
\hline
\end{tabular}

Time from epidural injection to first paresthesia sensation was similar between groups (Bupivacaine - $6.2 \pm 3.2 \mathrm{~min}$;
S75-R25-8.2 $\pm 6.2 \mathrm{~min}$ ). Types of sensations (heaviness, tingling, dumbness or increased temperature) were similar between groups and are shown in table III. Metameric sensory level at 5 minutes (T11 to T12), $10 \mathrm{~min}$ (T9), $15 \mathrm{~min}$ (T8). 20 $\mathrm{min}$ (T7 to T8) and $30 \mathrm{~min}$ (T7 to T8) were also similar between groups $(p>0.05)$.

Table III - Number of Patients in Each Group Referring Different Paresthesia Sensations in Lower Limbs after Epidural Local Anesthetic Administration

\begin{tabular}{lcc}
\hline & Group Bupivacaine & Group S75-R25 \\
\hline Numbness & $12^{*}$ & $17^{*}$ \\
Heaviness & 3 & 3 \\
Tingling & 2 & 1 \\
Increased temperature & 2 & 1 \\
\hline
\end{tabular}

${ }^{*} p<0.05$

As to blockade failure evaluated 30 minutes after epidural injection, there has been partial failure in one group S75-R25 patient and total failure in one patient, who needed intravenous sedation with propofol continuous infusion. No group Bupivacaine patient showed sensory failure $(p=0.2875)$. Motor block was more intense in group Bupivacaine as compared to group S75-R25 (Table IV) $(p=0.0117)$. Time for first postoperative analgesic request was longer for group S75-R25 as compared to group Bupivacaine (596 \pm 436 versus $463 \pm 270$ minutes, respectively), but visual analog scale values at this moment were similar between groups (Bupivacaine - $7 \pm 1.4 \mathrm{~cm}$; S75-R25 - $6.7 \pm 1.3 \mathrm{~cm}$ ).

Table IV - Motor Block Level Evaluated by the Anesthesiologist

\begin{tabular}{lcc}
\hline & Group Bupivacaine & Group S75-R25 \\
\hline Poor & $0 *$ & $2{ }^{*}$ \\
Good & $0 *$ & $3^{*}$ \\
Very Good & 4 & 4 \\
Excellent & 15 & 13 \\
\hline
\end{tabular}

${ }^{*} p<0.05$

As to perioperative adverse effects, 4 group Bupivacaine patients had arterial hypotension and were medicated with intravenous ephedrine; one patient had shivering, one patient had bradycardia not needing treatment, and one patient had nausea. In group $575-\mathrm{R} 25,3$ patients had arterial hypotension treated with intravenous ephedrine, one patient had shivering and the patient with blockade failure was submitted to intravenous sedation with propofol continuous infusion $(p>0.05)$. There were no postoperative adverse effects in both groups.

\section{DISCUSSION}

Literature reports that epidural administration of the isolated isomer $\mathrm{S}(-)$ bupivacaine (levobupivacaine) had a lower dose-dependent incidence of motor block and longer post-

Revista Brasileira de Anestesiologia Vol. 53, N² 2, Março - Abril, 2003 
operative analgesia 5,12 . Only $0.75 \%$ epidural levobupivacaine was similar to racemic bupivacaine in sensory block onset and motor block quality and duration ${ }^{13}$. The hypothesis that a formulation containing $25 \%$ of $\mathrm{R}(+)$ and $75 \%$ of S(-)bupivacaine would improve formulation's anesthetic profile was evaluated through the spinal route and both formulations were similar in anesthetic potency ${ }^{14}$.

However, results obtained in our study with epidural administration have shown that $25 \% \mathrm{R}(+)$ bupivacaine associated to $75 \% \mathrm{~S}(-)$ bupivacaine resulted in a less motor block intensity as compared to the racemic solution. A benefit has been longer postoperative analgesia with $\mathrm{S} 75-\mathrm{R} 25$ as compared to racemic bupivacaine. Other parameters evaluated, such as type of paresthesia sensation, sensory block failures, adverse effects of epidural administration and temperature were similar for both groups.

As compared to literature data, the mixture S75-R25 seemed to have the same behavior as pure levobupivacaine in terms of motor block ${ }^{5,10,11}$, while a higher $0.75 \%$ levobupivacaine concentration would be similar to the racemic formulation ${ }^{13}$. When $0.75 \%$ epidural levobupivacaine and $0.75 \%$ racemic bupivacaine were administered for lower abdomen procedures, motor block quality was evaluated by the surgeon and the anesthesiologist and, while surgeons considered both formulations identical, anesthesiologists were biased in quantifying racemic solution motor block as better than levobupivacaine, although without statistical significance ( $p$ $=0.07)^{13}$.

In our study, the same anesthesiologist was in charge of collecting motor block data which were then verified by a second co-author and were agreed upon by both. Although the anesthesiologist's classification of motor block as inferior (Bromage 2 and 3), the anesthetic technique was not impaired in $95 \%$ of group S75-R25 patients for the procedures being studied (saphenectomy and minor tibial orthopedic procedures). This study has not allowed us to conclude whether motor block quality would be impaired in intra-abdominal procedures.

The clinical interest in the search for new and more effective long-lasting anesthetic formulations resides in the fact that cardiovascular and central nervous system toxicity is seen with isomeric $R(+)$ bupivacaine ${ }^{1,4,5,15}$. The incidence of intoxication by local anesthetics is uncommon, however catastrophic. Local anesthetic drugs block axonal peripheral fast voltage-dependent sodium channels and these drugs have high affinity for cardiovascular and central nervous systems. Central nervous system toxicity is a two-step process which, in low concentrations, promotes inhibitory neurons block resulting in generalized seizures; in high concentrations, however, there is a global central nervous system depression. Whereas seizures are clinically impressive, they may in general be treated without permanent sequelae. Cardiotoxicity is the most important effect and may be indirectly mediated by the brain and directly mediated by the myocardium. Similarly to central nervous system, indirect cardiotoxicity is initially a stimulating action as a consequence of lower concentrations, and is followed by a de- pressing component in higher concentrations. Direct myocardial actions include negative inotropism, chronotropism and dromotropism. There is stereoselectivity for dromotropism. S(-) bupivacaine has caused less conduction delay as compared to the racemic formulation ${ }^{16}$. Lethal dose for $50 \%$ of animals (rats and mice) was $30 \%-40 \%$ lower for $\mathrm{R}(+)$ as compared to $\mathrm{S}(-)$ bupivacaine ${ }^{6}$. Cardiotoxicity is due to the fact that $\mathrm{R}(+)$ has more affinity and takes longer to dissociate from ion channels predisposing to arrhythmias ${ }^{4}$. In anesthetized dogs, mortality rate was $20 \%$ higher for the racemic formulation as compared to levobupivacaine ${ }^{17}$. The idea of having a long-lasting local anesthetic drug with good anesthetic and analgesic properties comparable to standard racemic bupivacaine, however with lower toxicity, is attractive. Alternatives deserving further evaluations would be the pure isomer $0.75 \%$ S(-) bupivacaine or S75-R25 in higher concentrations to maintain motor block quality and enhance analgesia. The association of the isomer $25 \% \mathrm{R}(-)$ bupivacaine could have theoretical benefits, such as more antibacterial potency against Staphylococcus aureus, $S$. epidermidis, Enterococcus faecalis ${ }^{18}$. It is still to be known whether the association of $25 \% \mathrm{R}(+)$ bupivacaine to the formulation would be more advantageous as compared to $0.75 \%$ levobupivacaine, being equipotent both in anesthesia and incidence of toxic effects.

Our data allow us to conclude that, in the conditions of this study, S75-R25 bupivacaine has induced prolonged analgesia duration and less intense motor block as compared to racemic bupivacaine (S50-R50).

\section{REFERÊNCIAS - REFERENCES}

01. Mather LE, Chang DH - Cardiotoxicity with modern local anaesthetics: is there a safer choice? Drugs, 2001;61:333-342.

02. Liguori GA, Chimento GF, Borow L et al - Possible bupivacaine toxicity after intra-articular injection for postarthroscopy analgesia of the knee: implications of the surgical procedure. Anesth Analg, 2002;94:1010-1013.

03. Garcia JBS, Oliveira JR, Silva EPA et al - Estudo comparativo entre levobupivacaína a 0,5\% e bupivacaína racêmica a $0,5 \%$ associadas ao sufentanil na anestesia peridural para cesariana. Rev Bras Anestesiol, 2001;51:377-384.

04. Ohmura S, Kawada M, Ohta T et al - Systemic toxicity and resuscitation in bupivacaine- levobupivacaine-, or ropivacaine-infused rats. Anesth Analg, 2001;93:743-748.

05. Foster RH, Markham A - Levobupivacaine: a review of its pharmacology and use as a local anaesthetic. Drugs, 2000;59: 551-579.

06. Casati A, Chelly JE, Cerchierini E et al - Clinical properties of levobupivacaine or racemic bupivacaine for sciatic nerve block. $\mathrm{J}$ Clin Anesth, 2002;14:111-114.

07. Glaser C, Marhofer P, Zimpfer G et al - Levobupivacaine versus racemic bupivacaine for spinal anesthesia. Anesth Analg, 2002;94:194-198.

08. Alley EA, Kopacz DJ, McDonald SB et al - Hyperbaric spinal levobupivacaine: a comparison to racemic bupivacaine in volunteers. Anesth Analg, 2002;94:188-193.

09. Ivani G, Borghi B, van Oven H - Levobupivacaine. Minerva Anestesiol, 2001;67:20-23. 
10. Vercauteren MP, Hans G, De Decker K et al - Levobupivacaine combined with sufentanil and epinephrine for intrathecal labor analgesia: a comparison with racemic bupivacaine. Anesth Analg, 2001;93:996-1000.

11. Murdoch JA, Dickson UK, Wilson PA et al - The efficacy and safety of three concentrations of levobupivacaine administered as a continuous peridural infusion in patients undergoing orthopedic surgery. Anesth Analg, 2002;94:438-444.

12. Delfino J, Vale NB - Bupivacaína levógira a $0,5 \%$ pura versus mistura enantiomérica de bupivacaína (S75-R25) a 0,5\% em anestesia peridural para cirurgias de varizes. Rev Bras Anestesiol, 2001;51:474-482.

13. Kopacz DJ, Allen HW, Thompson GE - A comparison of peridural levobupivacaine $0.75 \%$ with racemic bupivacaine for lower abdominal surgery. Anesth Analg, 2000;90:642-648.

14. Imbelloni LE, Beato L - Comparação entre bupivacaína racêmica (S50-R50) e mistura enantiomérica de bupivacaína (S75-R25), ambas Isobáricas a 0,5\% em raquianestesia. Estudo em cirurgias ortopédicas. Rev Bras Anestesiol, 2001;51:369-376.

15. Gristwood RW - Cardiac and CNS toxicity of levobupivacaine: strengths of evidence for advantage over bupivacaine. Drug Saf, 2002;25:153-163.

16. Graf BM - The cardiotoxicity of local anesthetics: the place of ropivacaine. Curr Top Med Chem, 2001;1:207-214.

17. Groban L, Deal DD, Vernon JC et al - Cardiac resuscitation after incremental overdosage with lidocaine, bupivacaine, levobupivacaine, and ropivacaine in anesthetized dogs. Anesth Analg, 2001;92:37-43.

18. Hodson M, Gajraj R, Scott NB - A comparison of the antibacterial activity of levobupivacaine versus bupivacaine: an in vitro study. Br J Anaesth, 1998;81:284.

\section{RESUMEN}

Gonçalves RF, Lauretti GR, Mattos AL - Estudio Comparativo entre Bupivacaína a 0,5\% y Mezcla Enantiomérica de Bupivacaína (S75-R25) a 0,5\% en Anestesia Peridural

Justificativa y Objetivos - La mezcla enantiomérica de bupivacaína (S75-R25) viene siendo empleada por su propiedad anestésica con menor toxicidad de que la bupivacaína racémica. El objetivo de este estudio es comparar la bupivacaína a $0,5 \%$ con la mezcla enantiomérica de bupivacaína a 0,5\% (S75-R25) en anestesia peridural.

Método - Fueron incluidos en el estudio 44 pacientes divididos en dos grupos $(n=22)$ denominados de Bupivacaína y S75-R25. Los pacientes fueron medicados con midazolam por vía venosa. La anestesia peridural fue realizada en el espacio $L_{3}-L_{4}$ ó $L_{2}-L_{3}, y$ administrado 16 a $24 \mathrm{ml}$ de la solución del anestésico local. El grupo Bupivacaína recibió bupivacaína a $0,5 \%$ con vasoconstrictor. El grupo $S 75-R 25$ recibió la mezcla enantiomérica de bupivacaína a $0,5 \%$ con vasoconstrictor. Fueron evaluados la temperatura del miembro inferior antes y después del bloqueo peridural, el tiempo de latencia del bloqueo, el tipo de alteración referida por el paciente, posibles fallas sensoriales, nivel sensorial metamérico y el grado de bloqueo motor. En la sala de recuperación pós-anestésica, fue anotado el tiempo de requisición del primero analgésico.

Resultados - Hicieron parte de la evaluación final 41 pacientes. Los grupos fueron demográficamente semejantes. La dosis per-operatoria de midazolam, el volumen de anestésico local por vía peridural, el tiempo de latencia para la instalación del bloqueo, fallas sensoriales la picada de la aguja, temperatura del miembro inferior en los diferentes tiempos, el tipo de sensación parestésica, y el nivel anestésico en dermátomos fueron semejantes entre los grupos. El grado de bloqueo motor fue más intenso para el grupo Bupivacaína, comparado al grupo S75-R25 ( $p=0,0117)$. El tiempo para requisición del primero analgésico en el período pós-operatorio fue superior para el grupo S75-R25, comparado al grupo Bupivacaína (596 \pm 436 minutos versus $463 \pm 270$ minutos, respectivamente; $p=0,04572$ ). La incidencia de efectos adversos fue semejante entre los grupos.

Conclusiones - La mezcla enantiomérica de bupivacaína (S75-R25) presentó mayor tiempo analgésico y menor grado de bloqueo motor, comparada con la solución de bupivacaína racémica. 